\title{
Cardiac Sodium Channel Blockade Due to Antiepileptic Drug Combination
}

\author{
Ossama Maadarani ${ }^{1}$, Zouheir Bitar ${ }^{1}$, Abdelaziz Ashkanani ${ }^{1}$, Mahmoud Elzoueiry ${ }^{1}$, Mohamad Elhabibi $^{1}$, Mohamad Gohar $^{1}$, \\ Mohamad Almuwaizri², Sania Shoeb³ ${ }^{3}$, Jadan Alsaddah ${ }^{4}$ \\ ${ }^{1}$ Internal Medicine Department, Ahmadi Hospital, Kuwait Oil Company, Al Ahmadi, Kuwait \\ ${ }^{2}$ Nursing Department, Ahmadi Hospital, Kuwait Oil Company, Al Ahmadi, Kuwait \\ ${ }^{3}$ Internal Medicine Department, Al Salam Hospital, Kuwait \\ ${ }^{4}$ Department of Cardiology, Chest Diseases Hospital, Kuwait
}

Received: 20/08/2021

Accepted: 26/08/2021

Published: 04/10/2021

\begin{abstract}
How to cite this article: Maadarani O, Bitar Z, Ashkanani A, Elzoueiry M, Elhabibi M, Gohar M, Almuwaizri M, Shoeb S, Alsaddah J. Cardiac sodium channel
\end{abstract} blockade due to antiepileptic drug combination. EJCRIM 2021;8: doi:10.12890/2021_002839.

Conflicts of Interests: The authors declare there are no competing interests.

This article is licensed under a Commons Attribution Non-Commercial 4.0 License

\section{ABSTRACT}

Drugs that inhibit voltage-dependent sodium channels are commonly used to treat epilepsy. Old and novel antiepileptic drugs are used either as monotherapy or in combination to control epilepsy. For a long time, carbamazepine has been used as the first choice for the treatment of simple and complex partial seizures. In the USA, lacosamide was approved in October 2008 as an adjunctive treatment for partial-onset seizures. We describe the effect of two sodium channel blockers on the heart of a patient with epilepsy.

\section{LEARNING POINTS}

- Approximately $30 \%$ of patients with epilepsy require combination therapy with antiepileptic drugs for seizure reduction.

- Lacosamide and carbamazepine are both sodium channel blockers but exert their effects through different mechanisms.

- Electrocardiogram monitoring is necessary when lacosamide and carbamazepine are used together as this combination may predispose to seizures, conduction abnormalities and dysrhythmia.

\section{KEYWORDS}

Sodium channel blockers, lacosamide, electrocardiogram

\section{INTRODUCTION}

Some anticonvulsant drugs may cause seizures and dysrhythmia if administered in too high a dose. Many drugs including antiarrhythmic and antiepileptic drugs (AEDs) act as sodium channel blockers. Lacosamide and carbamazepine are both AEDs which act through blockade of voltage-dependent sodium channels, but they exert their effects through different mechanisms. We present a case where a combination of two AEDs acting as sodium channel blockers led to tonic-clonic seizures and significant ECG changes similar to those seen with tricyclic antidepressants, and which required immediate intervention to prevent further seizures and lethal cardiac dysrhythmia.

\section{CASE DESCRIPTION}

A 68-year-old man with a known history of epilepsy, hypertension and atrial fibrillation was admitted to hospital with a 1-week history of recurrent seizures. His chronic medication included extended-release carbamazepine $400 \mathrm{mg}$ twice daily, bisoprolol 5 mg once daily and apixaban $5 \mathrm{mg}$ twice daily. The patient and his family members confirmed good compliance with medication. The patient's blood pressure was $130 / 80 \mathrm{mmHg}$ and he had an irregular pulse of $70-80$ beats per minute, a respiration rate of 20 breaths per minute and a normal 
temperature. Physical examination revealed an irregular heartbeat; the rest of the examination was unremarkable. Magnetic resonance imaging (MRI) of the brain showed no changes since the previous imaging. Laboratory tests revealed no significant deviations from normal reference values. His ECG (Fig. 1) showed controlled atrial fibrillation and right axis deviation. No clear cause for the recurrent seizures was found.

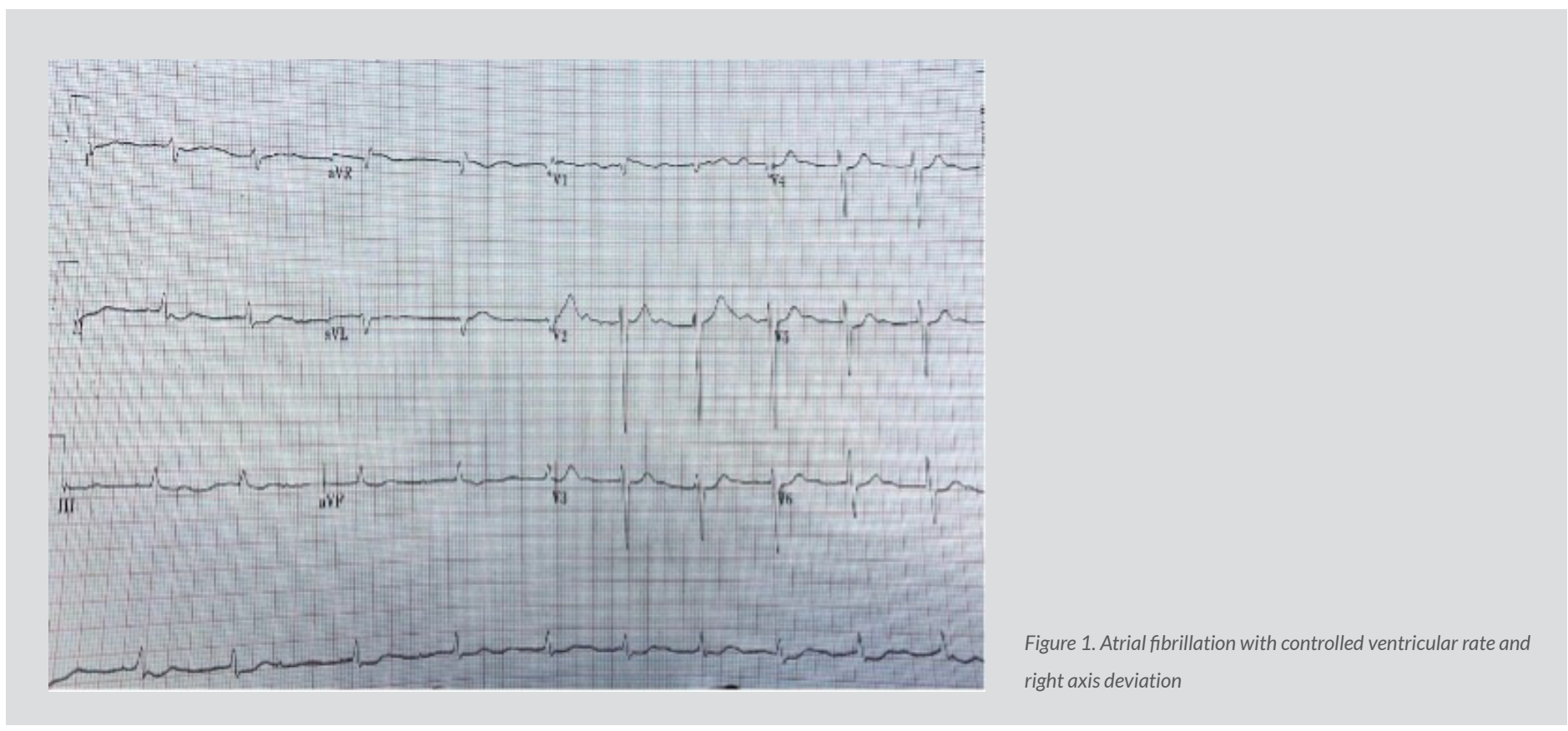

Lacosamide $50 \mathrm{mg}$ twice daily was started as adjunctive therapy in addition to the carbamazepine. Follow-up over the first week showed a significant reduction in seizures but the follow-up ECG demonstrated no changes. After 1 week, the dose of lacosamide was increased to 100 $\mathrm{mg}$ twice daily. After the second $100 \mathrm{mg}$ dose of lacosamide, the patient developed a generalized tonic-clonic seizure and ECG monitoring showed a wide QRS complex. Lorazepam $4 \mathrm{mg}$ was given intravenously to control the seizure and the airway was secured. The patient was transferred to the intensive care unit.

His ECG showed a regular rhythm with heart rate of 93 beat/min with very wide QRS complex of 0.24 ms and a dominant secondary R (terminal $R^{\prime}$ ) wave in lead AVR (Fig. 2). The picture of tonic-clonic seizure after the increased dose of lacosamide in combination with carbamazepine and significant changes on ECG, particularly the extreme widening of the QRS with a dominant secondary R wave in lead AVR, raised the possibility of sodium channel blocker (lacosamide and carbamazepine) toxicity similar to a tricyclic antidepressant overdose. The airway was secured with intubation and mechanical ventilation to maintain the $\mathrm{pH}$ above 7.50 through hyperventilation, and intravenous sodium bicarbonate was started with $100 \mathrm{mEq}$ boluses every 5-10 minutes with close monitoring of the QRS complex width on the monitor and ECG. Electrolytes were all within the normal range. Gradually the width of the QRS started to narrow and venous blood gases showed a pH of 7.51. After 2 hours, the follow-up ECG was almost back to baseline (Fig. 3) and the patient was extubated after a few hours. An alternative antiepileptic medication, levetiracetam, was started and lacosamide and carbamazepine were discontinued.

\section{DISCUSSION}

Research has showed that nearly two-thirds of patients with epilepsy require monotherapy with AEDs, with the other third needing a combination of AEDs ${ }^{[1]}$. To reduce seizure frequency and activity, the physician can select a combination of AEDs, taking into consideration the pharmacodynamics and possible pharmacokinetic interactions between them ${ }^{[2]}$. AEDs most commonly inhibit voltage-dependent sodium channels located on the cell membrane and alter the sodium current. For a long time, carbamazepine has been used as the first choice to treat simple and complex partial seizures. In the USA, lacosamide was approved in October 2008 as an adjunctive treatment for partial-onset seizures ${ }^{[3]}$. Depolarization of cardiomyocytes (atria and ventricles) and neurons depends on voltage-gated sodium channels creating and propagating action potentials, which suggests that blocking these channels with medication will result in the prolongation of depolarization in these excitable cells ${ }^{[4]}$. In concentration-dependent behaviour, both carbamazepine and lacosamide inhibit the cardiac sodium channel SCN5A ${ }^{[5]}$. 

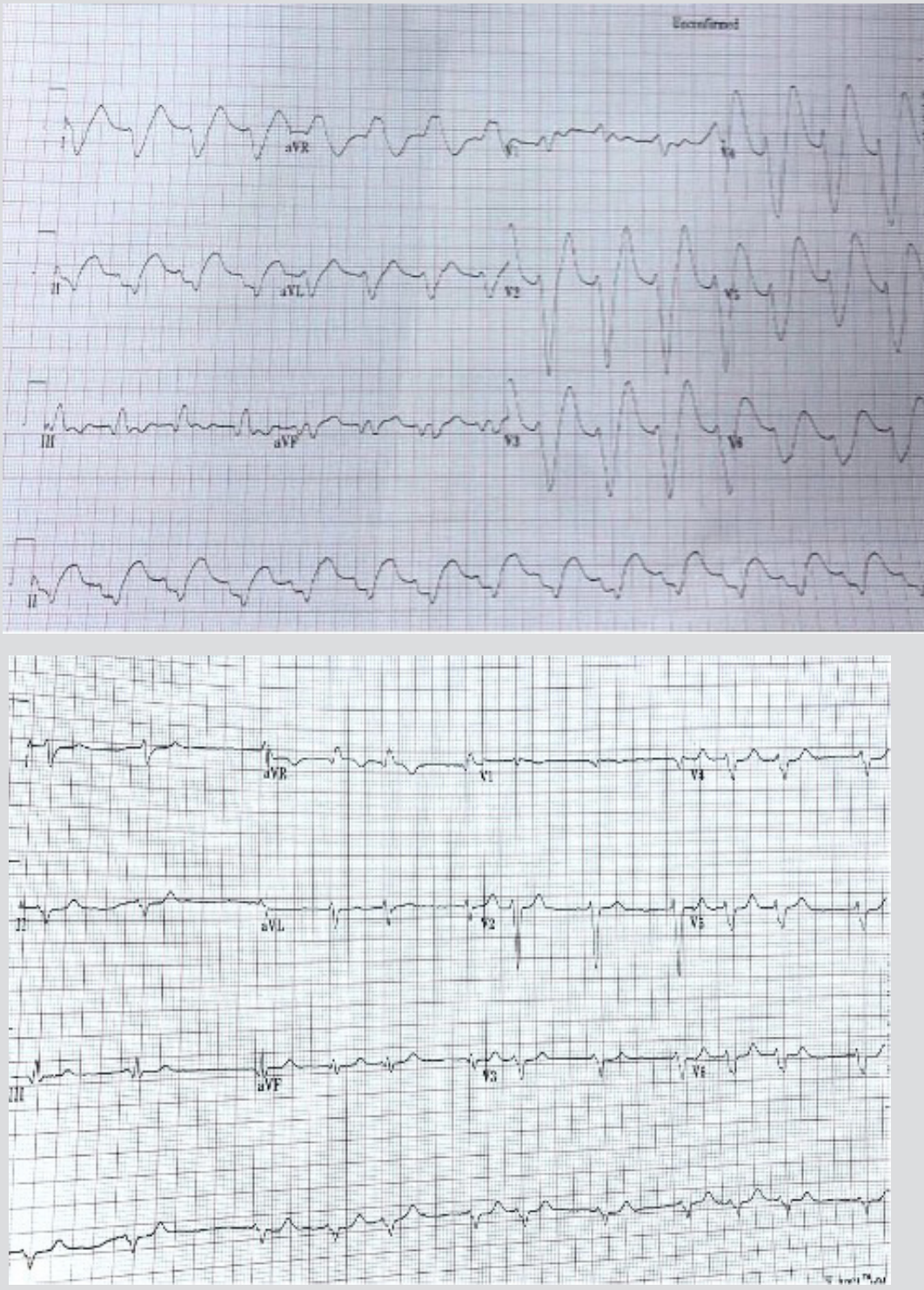

Figure 2. Significantly wide QRS complex of $0.24 \mathrm{~ms}$ and secondary R wave (terminal $R^{\prime}$ ) in lead AVR
Figure 3. ECG almost back to baseline after treatment with sodium bicarbonate

However, through its novel mechanism, lacosamide enhances and stabilizes the slow-inactivation of the voltage-gated sodium channels as opposed to other antiepileptic medications such as carbamazepine which exert their efficacy mainly on the fast-inactivation sodium channel[ ${ }^{[6]}$. This may explain the possible amplified cardiac effect when the dose of lacosamide is increased or it is added to other sodium channel blockers like carbamazepine.

There are limited data regarding cardiac arrhythmia and AEDs in the literature ${ }^{[7]}$. DeGiorgio et al. reported a case of ventricular tachycardia associated with lacosamide co-medication in drug-resistant epilepsy ${ }^{[8]}$. Another case report demonstrated conduction disturbances and arrhythmias related to lacosamide treatment ${ }^{[9]}$.

We report the case of a patient with epilepsy who developed tonic-clonic seizures and significant ECG changes similar to those seen in tricyclic antidepressant cardiac toxicity, when the dose of lacosamide, newly prescribed as an adjunctive therapy to baseline carbamazepine, was increased. The ECG changes included significant widening of the QRS complex to $0.24 \mathrm{~ms}$ in addition to a dominant secondary R wave (terminal R') in lead AVR, which is consistent with the side effects of sodium channel blockers on the conduction system of cardiomyocytes. Prolongation of the QRS complex above $100 \mathrm{~ms}$ is associated with seizures, while serious ventricular dysrhythmia may occur if the width of the QRS is greater than $160 \mathrm{~ms}$. In our patient, the combination of two AEDs acting as sodium channel blockers, namely lacosamide and carbamazepine, led to paradoxical tonic-clonic seizures and significant conduction abnormality on ECG that may predispose to serious ventricular arrhythmia. Treatment approaches in the setting of sodium channel blockade AED toxicity include benzodiazepines as the 
primary treatment for seizures, repeated doses of intravenous sodium bicarbonate with a goal of pH 7.45-7.55 and continuous monitoring of ECG to assess response. Hyperventilation in the intubated patient may also be utilized to further alkalinize serum. Fortunately, in our case, although the width of the QRS reached $0.24 \mathrm{~ms}$, no serious ventricular dysrhythmias were recorded, and the response to repeated intravenous sodium bicarbonate boluses showed improvement in QRS width. An alternative antiepileptic medication with a different mechanism, levetiracetam, was started and lacosamide and carbamazepine were discontinued. No more seizures were recorded.

\section{CONCLUSION}

The combination of two antiepileptic drugs which act as sodium channel blockers, such as lacosamide and carbamazepine, needs to be monitored with ECG since it may predispose to seizures, conduction abnormality and dysrhythmia.

\section{REFERENCES}

Kwan P, Schachter SC, Brodie MJ. Drug-resistant epilepsy. N Engl J Med 2011;365:919-926.

Brodie MJ. Pharmacological treatment of drug-resistant epilepsy in adults: a practical guide. Curr Neurol Neurosci Rep 2016;16:82.

Patsalos PN, Perucca E. Clinically important drug interactions in epilepsy: general features and interactions between antiepileptic drugs. Lancet Neurol 2003;2:347-356. Nelson CE, Hakim CH, Ousterout DG, Thakore PI, Moreb EA, Castellanos Rivera RM, et al. In vivo genome editing improves muscle function in a mouse model of Duchenne muscular dystrophy. Science 2016;351(6271):403-407.

5. Beyreuther BK, Freitag J, Heers C, Krebsfänger N, Scharfenecker U, Stöhr T. Lacosamide: a review of preclinical properties. CNS Drug Rev 2007;13:21-42.

6. Sheets PL, Heers C, Stoehr T, Cummins TR. Differential block of sensory neuronal voltage-gated sodium channels by lacosamide [(2R)-2-(acetylamino)- N-benzyl-3methoxypropanamide], lidocaine, and carbamazepine. J Pharmacol Exp Ther 2008;326:89-99.

7. Tomson T, Kenneback G. Arrhythmia, heart rate variability, and antiepileptic drugs. Epilepsia 1997;38:S48-51.

8. DeGiorgio AM, Dessoa TE, Lee L, DeGiorgio CM. Ventricular tachycardia associated with lacosamide co-medication in drug-resistant epilepsy. Epilepsy Behav Case Rep 2013;1:26-28.

9. Nizam A, Mylavarapu K, Thomas D, Briskin K, Wu B, Saluja D, et al. Lacosamide-induced second-degree atrioventricular block in a patient with partial epilepsy. Epilepsia 2011;52:153-155. 